\title{
Detection of very high energy gamma-ray emission from IC 310 by the MAGIC telescopes
}

\author{
Julian Sitarek \\ Author University of Łódź, PL-90236 Łódź, Poland \\ E-mail: jusi@kfd2.phys.uni.lodz.pl
}

\section{Fabio Zandanel, Francisco Prada}

Inst. de Astrofisica de Andalucia (CSIC), E-18080 Granada, Spain

E-mail: fabio.zandanel@gmail.com, fpradaliaa.es

\section{Saverio Lombardi}

Università di Padova and INFN, I-35131 Padova, Italy

E-mail: slombardepd.infn.it

and

\section{for the MAGIC Collaboration}

\author{
Andrii Neronov \\ SDC Data Center for Astrophysics, Geneva Observatory, Chemin d'Ecogia 16, 1290 Versoix, \\ Switzerland \\ E-mail: Andrii.Neronov@unige.ch
}

\section{Christoph Pfrommer}

HITS, Schloss-Wolfsbrunnenweg 33, 69118 Heidelberg, Germany

E-mail: christoph.pfrommer@h-its.org

\section{Anders Pinzke}

UC Santa Barbara, CA 93106, Santa Barbara, USA

E-mail: apinzke@physics.ucsb.edu

\section{Dmitri Semikoz}

APC, 10 rue Alice Domon et Leonie Duquet, F-75205 Paris Cedex 13, France

E-mail: semikoz@gmail.com

We report on the detection of very high energy emission ( $>300 \mathrm{GeV}$ ) from IC310 with the MAGIC telescopes. IC 310 is a head-tail radio galaxy located in the Perseus galaxy cluster, and has been recently detected by the Fermi-LAT instrument. The spectrum observed by the MAGIC telescopes is hard (spectral index $-2.00 \pm 0.14$ ). Strong hints of variability are seen in the data. We discuss the possible origin of the very high energy gamma-ray emission.

25th Texas Symposium on Relativistic Astrophysics - TEXAS 2010

December 06-10, 2010

Heidelberg, Germany 


\section{Introduction}

IC 310 is a nearby ( $80 \mathrm{Mpc}$, redshift $z=0.019$ ) head-tail radio galaxy located in the Perseus cluster, $0.6^{\circ}(\sim 1 \mathrm{Mpc})$ away from NGC 1275 , the cluster's central galaxy. This type of galaxies display a radio morphology consisting of a bright head, superimposed to the optical galaxy, and a fainter, elongated tail. In the standard explanation of those objects, the jets are bent (by the ram pressure or the the thermal pressure gradient of the intracluster medium) towards one direction creating the "head" structure and fan out at larger distances in a characteristic tail that extends over many tens to hundreds of kpc (see e.g. [5]). Interestingly, [14] showed that the X-ray emission in IC 310 may originate from the central AGN of the BL Lac-type object. Other observed characteristics of IC 310 suggest that it may also be a dim (weakly beamed) blazar [12]. The LAT instrument on board the Fermi satellite has recently detected IC 310 [11] with 5 photons above $30 \mathrm{GeV}, 3$ of which had energy above $100 \mathrm{GeV}$.

MAGIC (Major Atmospheric Gamma Imaging Cherenkov) is a system of two $17 \mathrm{~m}$ Imaging Atmospheric Cherenkov Telescopes (IACT) located at the Roque de los Muchachos, Canary Island of La Palma $\left(28^{\circ} \mathrm{N}, 18^{\circ} \mathrm{W}, 2200 \mathrm{~m}\right.$ a.s.l). Presently both telescopes work together in stereo mode at energies $\gtrsim 60 \mathrm{GeV}$, and provide an excellent sensitivity of $<1 \%$ of the Crab Nebula flux (C.U.) at energies above $250 \mathrm{GeV}$. In this proceeding, we present the results of observations of the Perseus cluster performed between 2008 and 2010 with the MAGIC telescopes which led to the detection of IC 310 [4].

\section{Observation and Analysis}

The MAGIC I telescope observed the Perseus cluster for a total of $94 \mathrm{~h}$ between November 2008 and February 2010 [3]. Since the end of October 2009 the MAGIC II telescope was also taking data, allowing the stereo analysis. Observations were performed in two pointing positions offset by $0.4^{\circ}$ from NGC 1275 . IC 310 was in the field of view at the angular distance of $0.25^{\circ}$ and $1^{\circ}$ respectively. Only the closer position was used for the analysis.

After the data quality check, we obtained $20.6 \mathrm{~h}$ of MAGIC stereo data taken between October 2009 and February 2010 (see [4] for details of the analysis). The top panel in Fig. 1 shows the so-called thet $a^{2}$ plot (squared distance between true and reconstructed source positions) with an excess of 106 events, corresponding to $7.6 \sigma$ significance (calculated according to the prescription by [7], Eq.17). The source was also detected in $27.5 \mathrm{~h}$ of mono data (taken between September

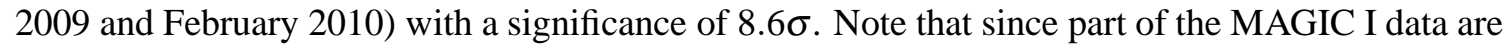
also used in the stereo analysis, the two significances are not completely independent.

It is interesting to note that contrary to $2009 / 2010$ data, the $11.2 \mathrm{~h}$ of good quality, mono data taken at the end of 2008 do not show any significant excess at the position of IC 310 (see Fig. 1, bottom right panel). The analysis of these data yield an upper limit for the flux $F(>300 \mathrm{GeV})<$ $2.35 \times 10^{-12} \mathrm{~cm}^{-2} \mathrm{~s}^{-1}$ corresponding to $1.9 \%$ C.U. (calculated using the [13] method with $95 \%$ of confidence level and assuming $30 \%$ systematic error in absolute flux level).

We also analyzed Fermi data taken during the period between 4th of August 2008 and 15th of July 2010 following the approach of [11]. We obtained the spectrum of IC 310 in the $2-200 \mathrm{GeV}$ energy band in two different ways. We performed a spectral fit using the standard Fermi unbinned 


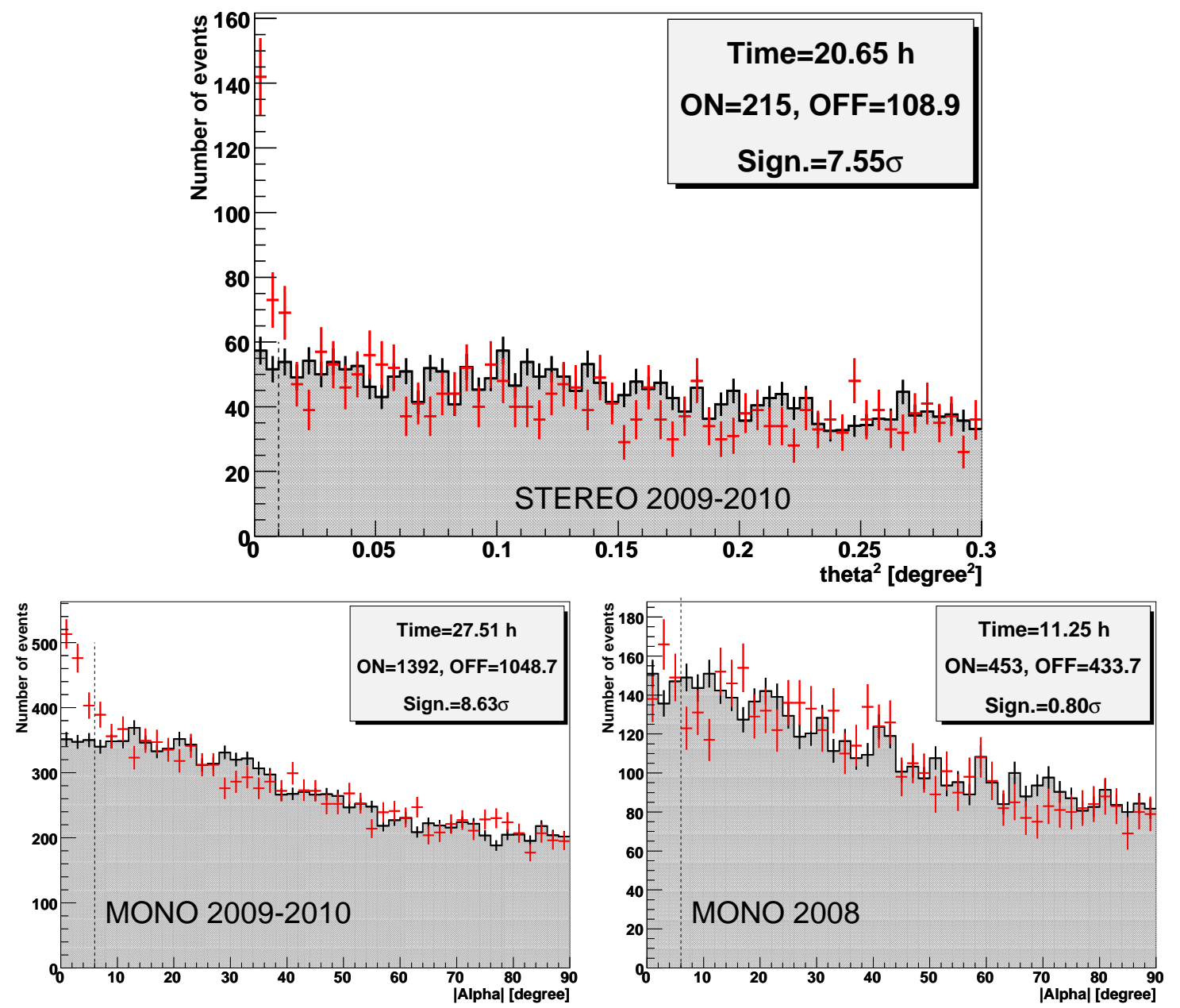

Figure 1: $T$ het $a^{2}$ distribution of the IC 310 signal and the corresponding background estimation from stereo observations performed between October 2009 and February 2010 (the top panel). alpha distribution from mono observations performed between September 2009 and February 2010 (bottom left), and NovemberDecember 2008 (bottom right). alpha and thet $a^{2}$ distributions are based only on the usage of the closer pointing position $\left(0.25^{\circ}\right.$ away from the IC 310$)$. The energy threshold of the analysis (defined as a peak of the differential MC energy distribution) is $260 \mathrm{GeV}$ for both mono and stereo analyses.

likelihood analysis, taking into account all sources from the $1^{\text {st }}$ year Fermi catalog [1] within $10^{\circ}$ of IC 310. We also obtain spectral points by extracting photon counts from the circle of radius $0.3^{\circ}$ centered on the source. Taking into account the proximity to a bright, nearby source (radio galaxy NGC 1275), we estimated the background in the Fermi analysis from three regions at $0.6^{\circ}$ away from NGC 1275 (see Fig. 2 and [11] for details).

\section{Results}

In Fig. 3, we show the MAGIC significance map of the Perseus cluster region above $400 \mathrm{GeV}$. The location of the bright spot is consistent with the position of the IC 310. In the panels inserted in Fig. 3, we also show historical VLA radio image of IC 310 [6]. The position of the gamma-ray 

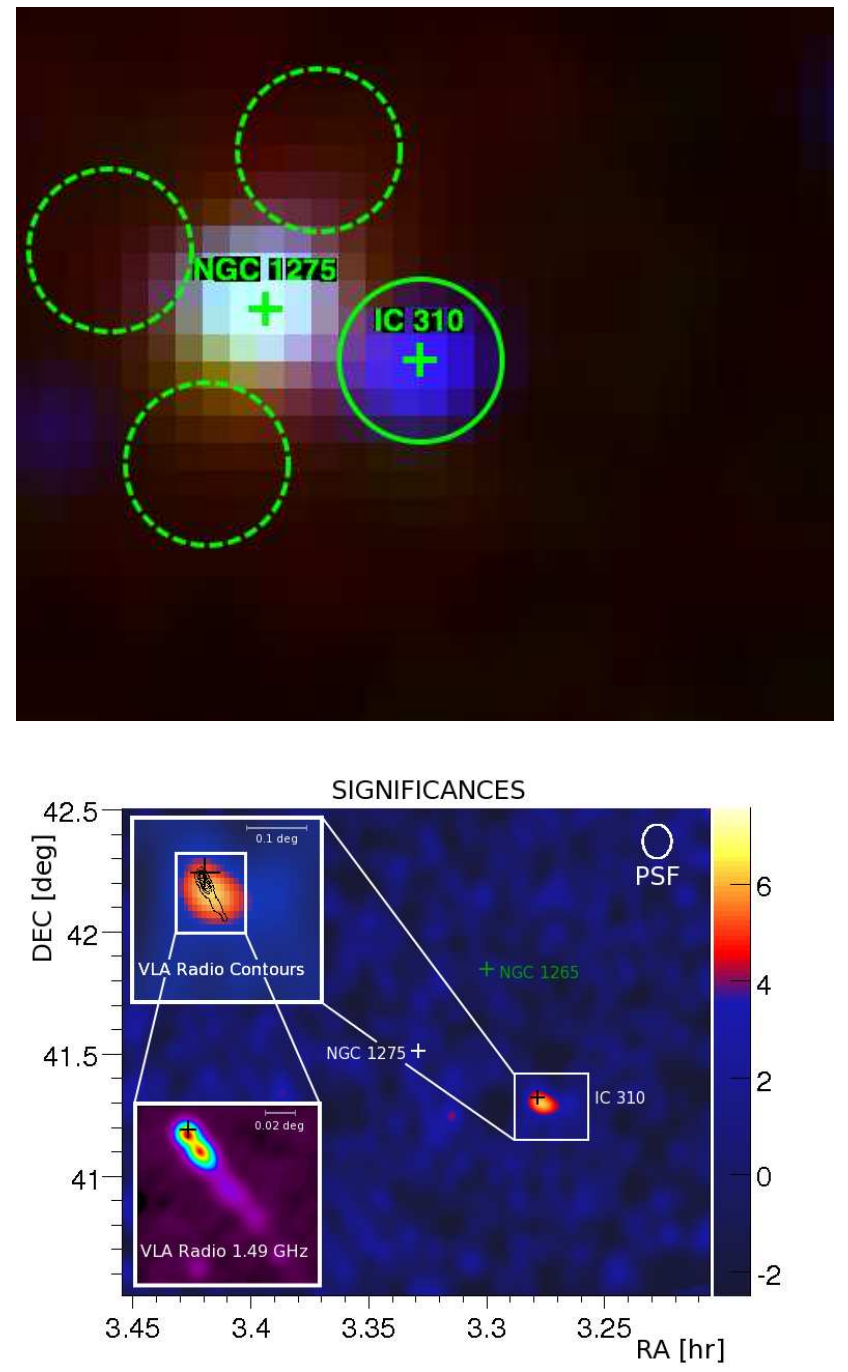

Figure 2: Fermi-LAT count rate image of the IC 310 region [4] in three energy bands: $0.3-3 \mathrm{GeV}$ (red), $3-30 \mathrm{GeV}$ (green) and $30-300 \mathrm{GeV}$ (blue). Solid (dashed) circles show the signal (background) integration regions used for obtaining the Fermi-LAT spectrum.

Figure 3: Significance skymap from the MAGIC stereo observation (42 h; both pointing positions, [4]) for energies above $400 \mathrm{GeV}$. In top left sub-panel we overlaid the IC 310 region with the NVSS (NRAO VLA Sky Survey at $1.49 \mathrm{GHz}$; [6]) contours. The corresponding NVSS image obtained with Aladin is shown in bottom left sub-panel. Positions of IC 310, NGC 1275 and NGC 1265 are marked with black, white, and green crosses respectively.

excess is coincident with the position of the radio emission. At energies above $400 \mathrm{GeV}$ the MAGIC stereo system has a point spread function (defined as a $40 \%$ containment radius, corresponding to the standard deviation of a 2-dimensional Gaussian distribution) of $\sim 0.06^{\circ}$, which does not allow to pinpoint the precise emission place.

The MAGIC stereo observations reveal a flat spectral energy distribution (SED) between $150 \mathrm{GeV}$ and $7 \mathrm{TeV}$ without any visible curvature or cut-off. The differential spectrum can be well fitted $\left(\chi^{2} / n_{d o f}=2.3 / 4\right)$ by a single power-law:

$$
d N_{\text {MAGIC }} / d E=(1.1 \pm 0.2) \times 10^{-12}(E / \mathrm{TeV})^{-2.00 \pm 0.14} \mathrm{~cm}^{-2} \mathrm{~s}^{-1} \mathrm{TeV}^{-1}
$$

The mean gamma-ray flux above $300 \mathrm{GeV}$ obtained from the stereo observations between October 2009 and February 2010 is $(3.1 \pm 0.5) \times 10^{-12} \mathrm{~cm}^{-2} \mathrm{~s}^{-1}$, corresponding to $(2.5 \pm 0.4) \%$ C.U. This value, combined with the upper limit from the 2008 data $(<1.9 \%$ C.U.) shows a hint of variability of IC 310 on a one-year time scale.

The Fermi-LAT points lay at the extrapolation of the MAGIC stereo points. Assuming a simple 

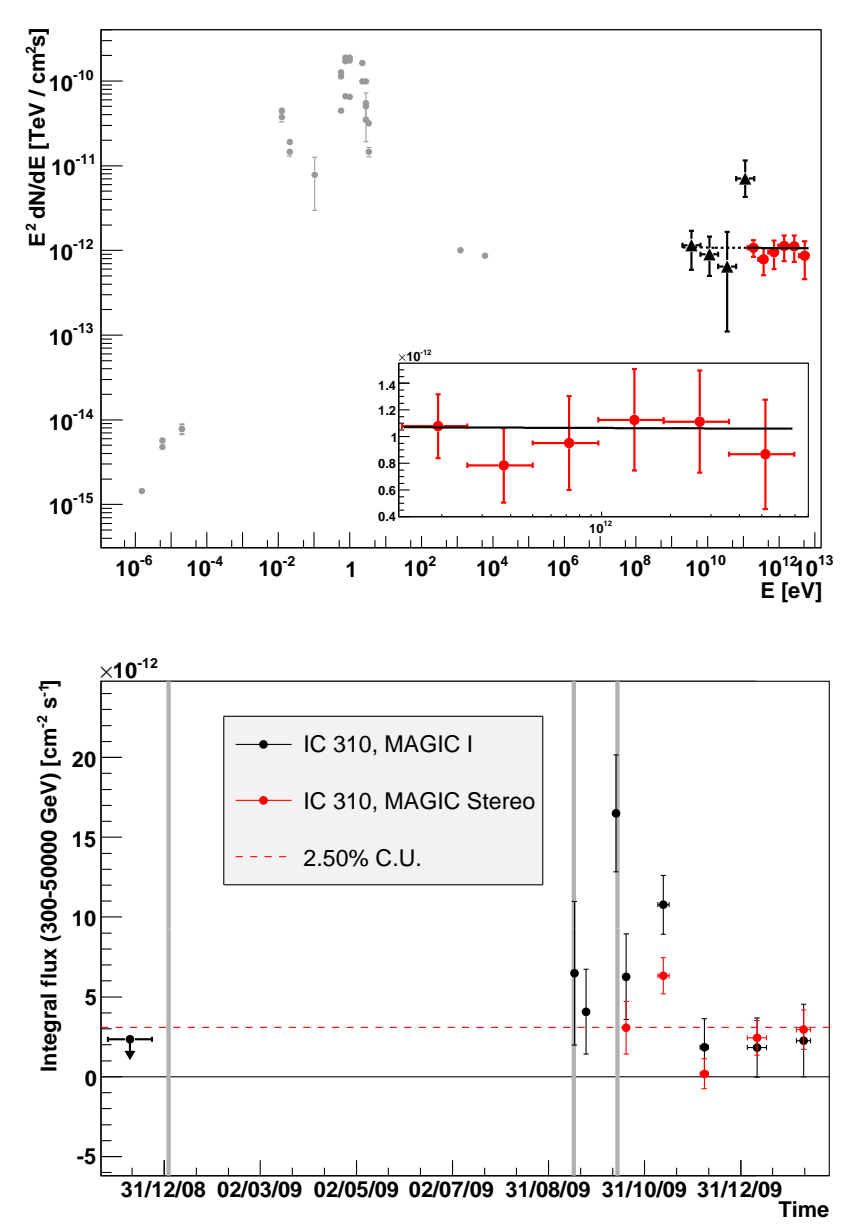

Figure 4: Spectral energy distribution of IC 310 obtained with $21 \mathrm{~h}$ of MAGIC stereo data (the red circles). Black triangles show the flux measurements from the Fermi-LAT instrument. Grey dots show historical X-ray, optical, IR and radio data obtained with NED database (see [4] and references within). The solid black line shows the spectral fit to the MAGIC data, and the dashed line the extrapolation to the $\mathrm{GeV}$ energy range. The inset shows a zoom of the MAGIC spectral points.

Figure 5: Light curve (in 10-day bins) of the gamma-ray emission above $300 \mathrm{GeV}$ obtained with MAGIC I (black) and MAGIC stereo (red) observations. The vertical grey lines shows the arrival times of the $>100 \mathrm{GeV}$ photons from the Fermi-LAT instrument [11]. The black circle with an arrow is the upper limit on the emission in November-December 2008. The horizontal red dashed line marks a flux level of $2.5 \%$ C.U.

power law for the Fermi spectrum, we obtain the differential flux in the energy range $0.15-7 \mathrm{TeV}$ :

$$
d N_{\text {Fermi }} / d E=(9.5 \pm 2.9) \times 10^{-9}(E / 10 \mathrm{GeV})^{-1.58 \pm 0.25} \mathrm{~cm}^{-2} \mathrm{~s}^{-1} \mathrm{TeV}^{-1}
$$

The Fermi-LAT spectral index is very hard, mostly due to the highest energy bin. Fermi-LAT and MAGIC stereo SED is showed in Fig. 4

Hints of variability can be seen in the MAGIC light curve. Fitting the individual light curves (see Fig. 5) assuming constant flux yields $\chi^{2} / n_{d o f}=27.6 / 7$ (for mono observations, corresponding to $3.5 \sigma$ ) and $17.5 / 4$ (stereo, $3.0 \sigma$ ). The largest deviations from the mean value are for the time intervals $13-14$ October 2009 ( $3.1 \sigma$ in mono above the mean flux), and $9-16$ November 2009 ( $3 \sigma$ in mono, $3.2 \sigma$ in stereo, above the mean flux).

\section{Conclusions and Discussion}

The strong indications of variability disfavour the gamma-ray production at the bow shock, discussed by [11]. In this case the emission should be steady on time scales of thousands of years. Assuming no Doppler boosting of the flux, variability with a time scale of a year (a week) constrains the transverse size of emission region to be $<10^{18} \mathrm{~cm}\left(<2 \times 10^{16} \mathrm{~cm}\right)$, which is much 
smaller than the size of the stationary shock $\left(\sim 10^{21} \mathrm{~cm}\right)$. The possible location of the gammaray emission region is in the innermost part of the jet (as e.g. for M87, see [2]). The combined MAGIC and Fermi spectral energy distribution is well described by a flat power law (index -2) over 3 orders of magnitude from $2 \mathrm{GeV}$ to $7 \mathrm{TeV}$. Such an extended, flat SED is hard to obtain in a simple one-zone SSC model (e.g. [9]). A viable model of emission might be inverse Compton scattering of external IR photon background photons from accretion flow or from the inner jet (see e.g. [10]). Alternatively, a flat spectrum can be produced in the hadronic models (e.g. [8]) or in more complicated, multi-zone leptonic models, where the $\mathrm{GeV}-\mathrm{TeV}$ emission of a few slightly shifted inverse Compton peaks can also emulate a flat spectrum (e.g. spine-sheath layer model, [15]).

\section{Acknowledgments}

We would like to thank the Instituto de Astrofísica de Canarias for the excellent working conditions at the Observatorio del Roque de los Muchachos in La Palma. The support of the German BMBF and MPG, the Italian INFN, the Swiss National Fund SNF, and the Spanish MICINN is gratefully acknowledged. This work was also supported by the Marie Curie program, by the CPAN CSD2007-00042 and MultiDark CSD2009-00064 projects of the Spanish Consolider-Ingenio 2010 programme, by grant DO02-353 of the Bulgarian NSF, by grant 127740 of the Academy of Finland, by the YIP of the Helmholtz Gemeinschaft, by the DFG Cluster of Excellence "Origin and Structure of the Universe", and by the Polish MNiSzW grant 745/N-HESS-MAGIC/2010/0.

\section{References}

[1] Abdo, A. A., et al. 2010, ApJS, 188, 405

[2] Acciari, V. A., et al. 2009, Science, 325, 444

[3] Aleksić, J., et al. 2010a, ApJ, 710, 634

[4] Aleksić, J., et al. 2010b, ApJL, 723, L207

[5] Begelman, M. C., Rees, M. J., \& Blandford, R. D. 1979, Nature, 279, 770

[6] Condon, J. J., \& Broderick, J. J. 1988, AJ, 96, 30

[7] Li, T.-P., \& Ma, Y.-Q. 1983, ApJ, 272, 317

[8] Mannheim, K. 1993, A\&A, 269, 67

[9] Maraschi, L., Ghisellini, G., \& Celotti, A. 1992, ApJL, 397, L5

[10] Neronov A., Aharonian F.A., 2007, ApJ., 671, 85

[11] Neronov, A., Semikoz, D., \& Vovk, I. 2010, A\&A, 519, L6

[12] Rector, T. A., Stocke, J. T., \& Perlman, E. S., 1999, ApJ, 516, 145 
[13] Rolke, W. A., López, A. M., \& Conrad, J. 2005, NIM A, 551, 493

[14] Sato, K., et al. 2005, PASJ, 57, 743

[15] Tavecchio, F., \& Ghisellini, G. 2008, MNRAS, 385, L98 